\title{
Geomechanical Assessment of the Effect of Inhomogeneities on the Propagation of Hydrofractures in Coal Seams
}

\author{
Mikhail Baev ${ }^{1, *}$, Veniamin Khyamyalyaynen ${ }^{1}$, and Aleksandr Shevtsov ${ }^{1}$ \\ ${ }^{1}$ T.F. Gorbachev Kuzbass State Technical University, Department of Theoretical and Geotechnical \\ Mechanics, 650000 Kemerovo, 28 Vesennya st., Russian Federation
}

\begin{abstract}
The article gives a theoretical propagation of the development of hydrofractures in coal seams from the position of geomechanics, including the possibilities and conditions for the fracture to intersect a natural crack. A technique is proposed for carrying out experimental studies of the effect of inhomogeneities on the extension of hydraulic fractures by physical modeling on samples of equivalent materials and the facility for their conduct. The results of experiments based on the proposed technique on samples made on the basis of cement, sand, fly ash, and water are presented, with modeling of inhomogeneities of various types. The results made it possible to conclude that when the closed cavities intersect in the form of gaping cracks of limited prevalence, their rupture fluid envelops with the preservation of the rupture plane. It is noted that when crossing unbounded gaping cracks, the established enveloping phenomenon will obviously occur along the "bridge" at the points of contacts of the opposite banks of cracks that take place in a real massif, and the very intersection of the fracture of the natural crack, mainly, depends on its deformation properties, the magnitude of the angle between the planes of the fracture and the crack, and also the fluid pressure.
\end{abstract}

\section{Introduction}

In the extraction of coal bed methane, the relationship between the production well and the natural network of cracks in the coal seam is crucial. This relationship directly characterizes the well drainage area of the CBM reservoir, and, consequently, the volume of produced gas. Traditionally, in order to create an artificial fracture intersecting the natural cleavage in coal seams, hydraulic fracturing technology is used [1-3]. In the process of implementation of this technology, a process fluid is injected into the isolated perforated interval of the well to form a new network of fractures in the coal seam and expand the existing cleavage, which in the next stage is filled with a propping agent (ceramic proppant or predominantly quartz sand) in order to preserve their conductive properties after stopping the injection. Nevertheless, the issue of the orientation of the created hydrofracture with regard to natural cracks in the coal seam is topical.

${ }^{*}$ Corresponding author: bma.gdk@gmail.com 


\section{Theoretical basis}

Let's consider hydraulic fracture cracking and fracture propagation in a homogeneous medium. Qualitatively, the hydraulic fracture cracking mechanism can be described on the basis of general positions of solid mechanics. To form an extended fracture in the rock mass, it is necessary to create a stress raiser in the form of fracture nucleus in it $[4,5]$. Then, injecting the fluid, the walls of the fracture should be loaded up to a pressure that would provide the appearance of destructive, tensile stresses on the fracture edges. Further fracture propagation in the rock mass is possible only due to an additional energy supply, which is ensured by the fluid velocity. In addition to the cost of further crack propagation, part of the fluid energy is expended on the deformation of the fracture walls (the fracture is extending from the well simultaneously with its propagation inward), the resistance to movement caused by the rheological characteristics of the fluid itself and the radius of the fracture, the filtration of the part of the fluid through the walls of the fracture [6-8]. Thus, it can be concluded that in a homogeneous medium with certain mechanical and filtration properties, the propagation of the fracture in a specified direction is completely determined by the technical characteristics of the injection means and the fluid properties.

Any rock mass is more or less inhomogeneous and anisotropic, has a block structure due to the presence of cracks in exogenous and endogenous genesis $[9,10]$. Therefore, in an inhomogeneous medium, apart from the above mentioned, the fracture propagation is also determined by the nature of its intersection with natural inhomogeneities in the form of cracks, different media interfaces, separate inclusions of materials with other properties, karst caverns, etc.

Let's consider the directional fracture propagation in a flexible rock mass in the presence of a natural crack with the opening $d$ in it. For the sake of simplicity, we consider a plane stress-strain state of a mass with the fracture orientation along the maximum of the principal stress components. Such a determination of the fracture plane direction is an obvious condition for its conservation in the course of the fracture propagation [11-13]. As a condition for the fracture propagation, we assume the condition for reaching the ultimate tensile stresses at the tips of the fracture. In this case the A. Griffith's energy fracture criterion and the critical value of tensile stresses established by him can be taken as a criterion of strength. As the conditions for the intersection of the fracture with the natural crack, we assume the following physical premises. When the fracture approaches the natural crack due to deformation of the mass under the action of fluid pressure, the opposite edges of the natural crack should be closed without getting a fluid into it. Let's suppose that when the natural crack closure occurs in uniaxial compression conditions and it should resist a fault to be intersected. Then, as a criterion of strength, the modified G.P. Cherepanov shear strength can be taken. Thus, as the conditions for the intersection of the fracture with the natural crack, we take the closure of the opposite edges and the shear resistance.

The calculation scheme, which explains the fracture propagation and its intersection with the natural crack, is shown in Fig. 1, where $P(r)$ is the distribution of the pressure fluid in the fracture; $\sigma_{r}, \sigma_{z}$ are the components of the principal stresses in the cylindrical coordinates $r, \varphi, z ; \sigma_{r 1}, \sigma_{z 1}$ are the components of the principal stresses in the coordinates $r_{1}$, $\varphi, z_{1} ; L$ is the distance from the well to the natural crack; $l$ is the characteristic size of the rock block in the out-of-plane direction from the fracture. Then the components of the rock block deformations can be represented in the following form:

$$
\varepsilon_{z}=\frac{1}{E}\left[\sigma_{z}-\mu\left(\sigma_{r}+\sigma_{\varphi}\right)\right]
$$




$$
\varepsilon_{r}=\frac{1}{E}\left[\sigma_{r}-\mu\left(\sigma_{\varphi}+\sigma_{z}\right)\right]
$$

where $\mu$ - Poisson ratio; $E$ - modulus of elasticity.

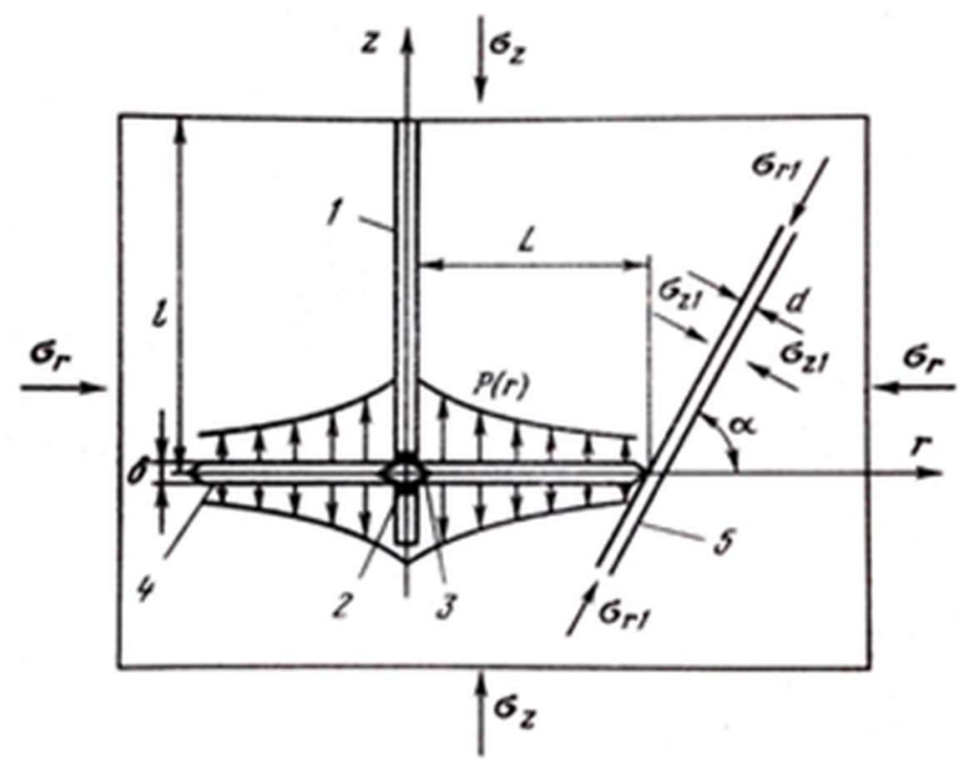

Fig. 1. The calculation scheme of the evaluation of the fracture propagation in an inhomogeneous medium: 1 -well; 2 -double packer; 3 - fracture nucleus; 4 - fracture; 5 -intercepted natural crack.

The critical value of the absolute value of tensile stresses at the tip of the fracture

$$
\left|\sigma_{z}-P(L)\right|=\left[\frac{2 E T}{\pi\left(1-\mu^{2}\right) L}\right]^{1 / 2}
$$

where $T$ - specific surface energy.

The criterion of strength at natural crack resistance to shear

$$
(1-a) \sigma_{c}=\left(\sigma_{r 1}-\sigma_{z 1}\right)-a\left(\sigma_{r 1}+\sigma_{z 1}\right),
$$

where $\sigma_{c}$ - ultimate compression strength; $a=f\left(1+f^{2}\right)^{1 / 2} ; f$ - friction ratio.

The fracture opening capacity is determined according to the formula (1)

$$
\delta=\int_{0}^{l}\left(\varepsilon_{z}-\varepsilon_{z}^{0}\right) d z=\frac{P(r)}{E} l .
$$

The natural crack opening curve is calculated by the expressions (1) and (2)

$$
d=\int_{0}^{l}\left(\varepsilon_{z}-\varepsilon_{z}^{0}\right) d z \cos \alpha+\int_{R_{W}}^{R}\left(\varepsilon_{r}-\varepsilon_{r}^{0}\right) d r \sin \alpha=\int_{0}^{l} \frac{P(r)}{E} d z \cos \alpha+\int_{R_{W}}^{R}-\frac{\mu P(r)}{E} d r \sin \alpha,
$$


where $\varepsilon_{z}^{0}$ and $\varepsilon_{r}^{0}$ - the components of the rock block deformations under natural stressstrain state, and the influence of the fluid pressure distribution of $P(r)$ on $\sigma_{r}$ is not taken into account.

To determine the fluid pressure in the fracture $P(r)$, let's consider the plane-radial flow in the fracture. Then, considering together the equations of motion and continuity, we obtain the following equation

$$
\frac{1}{r} \frac{d}{d r}\left[r \frac{k(r)}{\mu_{f}(r)} \frac{d P}{d r}\right]=0
$$

where $k(r)=\alpha^{*} \delta^{2} / 12$ - the fracture permeability index; $\alpha^{*}<1$ - constant taking into account the state of the fracture edges; $\mu_{f}(r)$ - the fluid dynamic viscosity coefficient.

Taking into account formula (5), the equation (7) is given by

$$
P\left(\frac{1}{r}-\frac{1}{\mu_{f}} \frac{d \mu_{f}}{d r}\right) \frac{d P}{d r}+2\left(\frac{d P}{d r}\right)^{2}+P \frac{d^{2} P}{d r^{2}}=0 .
$$

If $\mu_{f}=$ const, then the solution under the boundary conditions $P=P_{w}, d P / d r=B$ is given by

$$
P=\left(P_{w}^{3}+3 B P_{w}^{2} R_{w} \ln \frac{r}{R_{w}}\right)^{1 / 3}
$$

It follows that the distribution of the fluid pressure in the fracture does not depend on the deformation properties of the rock block, but is determined by the change in the fluid viscosity and, obviously, by the boundary conditions. At the well, it is necessary to set the injection pressure and the pressure gradient, that is, the fluid flow rate. The fracturing pressure $P(L)$ determined by formula (3) should be exerted on the fracture edges.

For a qualitative assessment of the possibility and the conditions for the intersection of the fracture with the natural crack, the $d$ value was calculated by formula (6). In this case, the equality of the obtained value to the crack opening value is taken as the condition for the natural crack closure. The hypothesis was made about the slow fracture propagation. Then the hydrodynamic resistance to fluid motion can be neglected, and the value $P=P(R)$ $=$ const can be taken as the solution of equation (8). It is taken that $\sigma_{r}=\sigma_{z}=\gamma H=2.5 \cdot 200=$ $5 \mathrm{MPa}, \sigma_{c}=70 \mathrm{MPa}, P=P(R)=13 \mathrm{MPa}, E=0.2 \cdot 10^{4} \mathrm{MPa}$. The results of the calculations are shown in Fig. 2, where the positive $d$ values correspond to the increase in the intersected crack opening, and the negative values correspond to the decrease, i.e., compression. It follows that the region of positive $d$ values corresponds to the further fracture propagation along the natural crack, and the region of negative values corresponds to its intersection.

It is easy to verify that for $\alpha$ values that correspond to compression, condition (4) is satisfied with a significant margin. The above calculation results can be used to determine the approximate values of the angles of the fracture crack intersection with the intersected crack $\alpha$ and its opening $d$, which ensure the propagation of the fracture in the specified direction. It should be noted that taking into account the hydrodynamic resistance to fluid motion in the fracture leads to an increase in the pressure $P$ and, consequently, to the expansion of the compression region of the intersected crack. 


\section{Experimental Methods}

Since an exact analytic solution of the problem of the fracture propagation in an inhomogeneous medium is not possible, an assessment was made of the effect of inhomogeneities on the fracture propagation in a rock mass by physical modeling on the equivalent material samples. The procedure for carrying out the experiments was as follows.

The tests were carried out on a laboratory installation (Fig. 2), which includes a sample and a system of fluid injection into the well model. Samples were made by pouring a hardening mixture produced on the basis of cement, sand, fly ash, and water into molds measuring $0.2 \times 0.2 \times 0.2 \mathrm{~m}$. The properties of the obtained material were controlled by the ratio of the mixture constituents and the time of its hardening before testing. The well was modeled with a hole of $0.02 \mathrm{~m}$ diameter. In the bottom-hole part of the well, the fracture nucleus - the $0.04 \mathrm{~m}$ diameter stress riser - was created. The fracture nucleus was created at the initial stage of the sample material hardening with a specially made laboratory cracker.

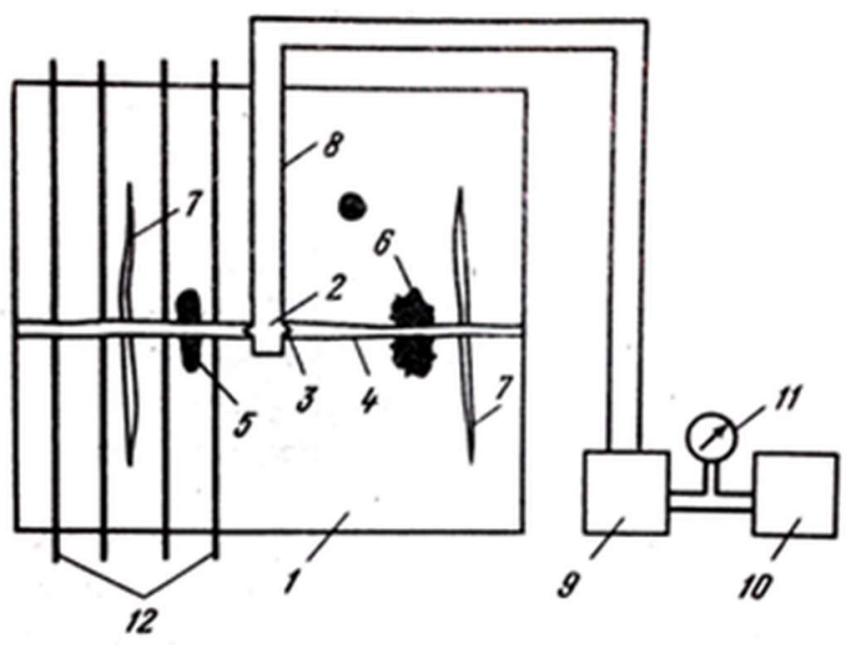

Fig. 2. Scheme of a laboratory installation for the oriented fracturing study on samples: 1 - sample; 2 - well model; 3 - fracture nucleus; 4 - fracture; 5 -inhomogeneity in the form of a smooth inclusion with a smooth surface; 6 - the same with a non-smooth surface; 7 -inhomogeneity in the form of an open crack; 8 - well casing (glued with epoxy resin); 9 - hydraulic accumulator; 10 - pump; 11 manometer; 12 - electrical sensors (graphite rods).

The natural inhomogeneities of the mass were modeled by unclosed unfilled cracks; cracks filled with solid and liquid paraffin; metal plates; separate inclusions in the form of expanded clay and quartz gravel; paper of different thickness and density. When using paraffin, a method for inhomogeneities modeling has been developed, including the preliminary fabrication of the crack frameworks by pouring molten paraffin into a special mold. In the process of making the samples, the cooled solid paraffin framework was placed in a mold and filled with a hardening mixture. Before the test, a sample was placed in a muffle furnace and heated to the melting point of paraffin. Thus, the modeling of the mass heterogeneity in the form of cracks filled with natural groundwater was achieved. Fracturing was carried out by water injecting with a pump at a minimum feed rate of 
$0,25 \cdot 10^{-3} \mathrm{~m}^{3} / \mathrm{s}$. The fracture propagation was fixed by the time of the triggering of the sensors (graphite rods) with the help of a special impulse counter. A total of 32 samples were tested. Some results are shown in Fig. 3.

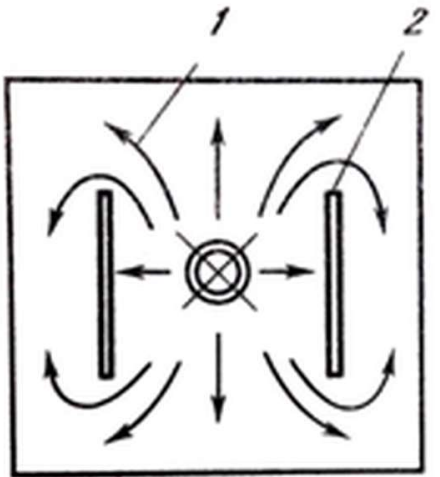

(a)

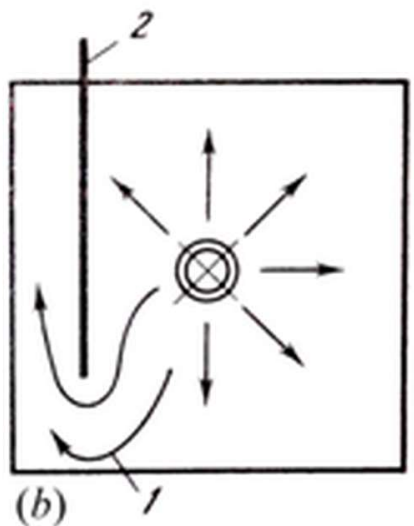

(b)

Fig. 3. The results of tesing the samples with inhomogeneities in the form of an open crack $(a)$ and a metal plate (b): 1 - fluid flow lines; 2 - inhomogeneity.

\section{Results}

On the basis of the results of the experiments conducted, let's consider in detail the mechanism of interaction of the fracture with the inhomogeneities of different types.

In all experiments with separate inclusions with an uneven surface, an "ideal" intersection of both unstable and strong inclusions occurred in a plane specified by fracture nucleus. In experiments with smooth surface inclusions, the intersection occurred, as a rule, along this surface without violating the integrity of the inclusion itself. In this case, the total sample fracture surface was maintained in the plane specified by fracture nucleus. It was found that the intersection of the separate inclusions with the fracture depends not on their mechanical properties, but on frictional forces and cohesion at the interface of the mediuminclusion system. Under high friction and adhesion, the fracture intersects the inclusion, and in the case of low friction, the fracturing occurs along the interface.

The fracture propagation through the open crack was studied on the above described homogeneous material samples containing a crack not opening on the surface of the sample with an opening of $0.001-0.002 \mathrm{~m}$. The crack in the sample was located perpendicular to the plane of fracture parallel to one of the lateral faces of the sample. In some samples, the cracks were filled with molten or solid paraffin. The results of the most typical tests of samples are shown in Fig. 4, a. A similar result was obtained in the presence of a metal plate instead of a crack in a sample (Fig. $4, b$ ). In all the cases considered, fracturing of a sample occurred strictly in the plane specified by fracture nucleus. The results of the series of experiments made it possible to conclude that, when intersecting with closed caverns in the form of open cracks of limited propagation or karst caverns, they are bent around by the fracturing fluid while maintaining the fracture plane. When intersecting the unlimited open cracks, it was found that bending phenomenon would obviously occur along the "bridge" at the contact points of the opposite edges of cracks that take place in a real rock mass.

It is found that even in a homogeneous medium (conditionally homogeneous), the fracture propagation occurs not along concentric circles, but along a certain helix around the well. 


\section{Discussion}

Thus, as a result of the studies carried out, it has been found that both in the presence of various solid inclusions and open caverns, there are all prerequisites for the directed fracture propagation in the oriented hydraulic fracturing. The revealed allows presenting the mechanism of the interception or the fracture with the natural cracks existing in a mass in the form of three stages. At the first stage, the fracture propagation occurs in a homogeneous medium. As a result of constant additional energy supply under the action of tensile stresses, the process of the fracture propagation into the interior of the mass proceeds with simultaneous increase in its opening. In this case, the tip of the fracture is ahead of the fluid propagation front and is sufficiently far from the natural crack. At the second stage, the tip of the fracture approaches the natural crack. Due to an increase in the stress concentration in the direction perpendicular to the plane of the fracture, there occur the mass compression and, as a consequence, depending on the intersection angle $\alpha$, either an over-compression or an increase in fracture opening. Under an over-compression, the further fracture propagation depends on the adhesion and friction force between the opposite edges of the fracture. In the presence of perfectly smooth edge surfaces, similar to our experiments with inhomogeneities in the form of separate solid inclusions with smooth surfaces, the fracture will change its direction and will propagate along the natural crack. However, in a real mass, natural cracks exhibit a certain tortuosity, wedge shape, the surfaces of their opposite edges are rather rough. Therefore, there is every reason to assert that, under the fracture over-compression, the adhesion and friction forces and at the interface of the opposite edges will be significant and the fracture will intersect with the natural crack similarly to the experiments with inhomogeneities in the form of separate solid inclusions with uneven surfaces. It should be noted that similar conclusions about the influence of the friction force on the intersection of the fracture with the interface between two different materials are obtained as a result of laboratory experiments given in $[14,15]$. At the third stage, the fracture intersects with the natural crack, and as it were, a "new" fracture nucleus is formed. There is a further increase in its opening and propagation inward. And the whole further process of the fracture propagation is repeated cyclically. In this case, the presence of natural cracks leads only to a decrease in the rate of the fracture propagation. The propagation of each natural crack stops allowing its sufficient expanding, and thus ensuring a good cohesion between the opposite edges of the crack.

Consequently, when the fracture approaches to the open cavern located out of fracture plane, it bends it around the perimeter or along the "bridges" (for example, along the natural rocky contacts between the opposite edges of the cracks) or by over-compressing this cavity with considerable adhesion of its opposite edges. In a real rock mass, these two processes seem to proceed simultaneously.

The possibility of the directed fracture propagation in the plane specified by the fracture nucleus is also confirmed by the results of field experiments on directed hydraulic fracturing of sandstone in underground mine conditions. In nature, the confirmation of the possibility of directed intersection with natural inhomogeneities is veins and dikes. In the process of formation, as a result of the movement of mineral substances from the lower layers of the earth's crust to the upper, they directionally intersected with the surrounding rocks with various mechanical properties and jointing.

\section{Conclusion}

Let's note the following. The intersection of the fracture with the natural rock mass crack mainly depends on its deformation properties, the magnitude of the angle between the planes of the facture and the natural crack, and also the fluid pressure. 
The nature of the intersection of the fracture with the interface between two media with different mechanical properties is largely independent of these properties, but is determined by the adhesion and friction between the interfacing surfaces. In the presence of surfaces that are close to perfectly smooth, further fracture propagation occurs along the interface. With increasing adhesion and friction between the contacting surfaces, the fracture intersects with the interface at some angle to the fracture plane specified by fracture nucleus. The magnitude of the angle depends on the adhesion and friction: the larger they are, the smaller the angle is.

The intersection with the open cavities in the form of caverns in a rock mass occurs by bending them around with the fluid while maintaining the direction of the fracture plane given by specified by the fracture nucleus.

\section{References}

1. M. Economides, L. Watters, S. Dunn-Norman, Petroleum well construction (Wiley, Duncan, 2000)

2. R. Rogers, K. Ramurthy, G. Rodvelt et al., Coal bed methane: principles and practices (Starkville, Oktibbeha, 2007)

3. M. Economides, T. Martin, Modern fracturing. Enhancing natural gas production (ET Publishing, Houston, 2007)

4. B. Brady, J. Elbel, M. Mack, et al., Oilfield Review, 2, 4 (1992)

5. T. Anderson, Fracture mechanics. Fundamentals and applications (CRC Press, Boca Raton, 2005)

6. K. Hubbert, D. Willis, Petroleum Transactions, 210, 153 (1956)

7. R. Hertzberg, Deformation and fracture mechanics of engineering materials (John Wiley \& Sons, Hoboken, 1996)

8. E. Fjar, et al., Petroleum related rock mechanics (Elsevier Science, Amsterdam, 2008)

9. C. Barton, M. Zoback, Geological Applications of Well Logs: AAPG Methods in Exploration, 13, 229 (2002)

10. T. Bai, Z. Chen, S. Aminossadati, et al., Journal of Petroleum Science and Engineering, 159, 257 (2017)

11. J. Rice, Fatigue Crack Propagation, 415, 247 (1967)

12. T. Golf-Racht, Fundamentals of fractured reservoir engineering (Elsevier Scientific Publishing Company, New York, 1982)

13. M. Zoback, Reservoir geomechanics (Cambridge University Press, New York, 2007)

14. M. Hanson, R. Shaffer, G. Anderson, Society of Petroleum Engineers, 4, 435 (1981)

15. T. Li, J. Wan, The Open Petroleum Engineering Journal, 9, 247 (2016) 